\title{
Anti-Inflammatory Dietary Supplements in Prevention of Diseases in Geriatric People
}

\author{
Shrihari TG* \\ Department of Oral Medicine \& Radiology, Krishnadevaraya Dental College and hospital, India \\ *Corresponding author: Shrihari TG, Assistant Professor, Department of Oral Medicine \& Radiology Krishnadevaraya dental college and hospital, \\ Bangalore -562157, Karnataka, India \\ Submission: 制September 11, 2017; Published: 眥January 19, 2018
}

\begin{abstract}
As our age advances, many changes are seen in our body, such as cellular changes, cardiovascular problems, cerebrovascular diseases, including cancer mediated by inflammation and their mediators such as free radicals (ROS,RNS), Cytokines, Transcription factors (NF-KB, STAT3) due to altered dietary patterns and digestive disorders. The disease pattern can be suppressed by including anti-inflammatory dietary supplements in our diet to prevent various diseases in geriatric peoples associated with inflammation. This article briefs about anti-inflammatory dietary supplements in prevention of diseases associated with inflammation in geriatric people.
\end{abstract}

Keywords: Free radicals; NF-KB; IL-1ß; TNF- $\alpha$; STAT3; COX2; Anti-oxidants; Vitamin A; Vitamin C; Vitamin E; Vitamin D; Omega 3 fatty acids

\section{Introduction}

Most of all cancers are preceded by chronic inflammation is the body's response to any type of injury in the form of physical or chemical or thermal or infectious agents. Inflammation is an initial step to many type of diseases namely cardiovascular diseases such as Angina Pectoris, Myocardial Infarction, Neurovascular diseases such as Parkinsonism and Alzheimer's diseases. And, 25\% of cancers are preceded by chronic inflammation such as oral cancer associated with oral potentially malignant lesions and conditions, Crohn's diseases, Prostate cancer, Breast cancer, cervical cancer, and Head and Neck cancer. Chronic inflammation is also considered as seventh hallmark of cancer [1-5].

\section{Anti-inflammatory dietary supplements in prevention of diseases in geriatric people}

Geriatric patients are prone to many diseases because of altered dietary patterns, reduced immune activity, altered cell proliferation and regeneration, muscle wasting, altered digestive pattern, and bone changes that lead to various complications.

Inflammation is preceded by most of all diseases. Hence, our aim is to suppress the inflammation by dietary supplements to prevent diseases for better health of an individual in geriatric peoples. Anti-inflammatory dietary supplements present in the food are Vitamin A, Vitamin C, vitamin E, Omega 3 fatty acids, Vitamin D, probiotics, curcumin [2].

Curcumin is apolyphenol, active component of Turmeric, extracted from the dried Rhizome of the Curcuma Longa plant. It has an anti-inflammatory property by down regulating key transcription factors of inflammation are NF-KB and STAT-3 mediated regulation of IL-1, IL-6, IL-8, TNF- $\alpha$, 5-LOX, CXCR-4,COX-2, iNOS pro -inflammatory genes involved in various inflammatory reactions and cancer [2,6].

Daily recommended dosages: $3 \mathrm{mg} / \mathrm{kg}$ body weight. $0.5 \mathrm{gm}$ and 7.5gm Curcumin/day- divided into 3-4 even doses.

Sources: Made from the Rhizomes of the plant Curcuma Longa.

Vitamin A, Vitamin C, Vitamin E are a vitamin, which neutralize the release of free radicals such as ROS, and RNS involved in oxidative stress induced cytokine activation and transcription factor that promotes DNA damage, cell aging, and cell death. They prevent the cell aging and cell death by reduction of inflammation associated with inflammatory conditions and cancer $[1,9,10]$.

Daily recommended dosage of vitamin A is $900 \mathrm{mg} /$ day.

Sources: Diary products, fish, dark chocolate's, fruits and vegetables such as tomatoes and papaya.

Daily recommended dosage of Vitamin C is $90 \mathrm{mg} /$ day.

Sources: Citrus fruits such as orange, peppers, Dark leafy vegetables, Tomatoes, Broccoli, Papaya, Green peas.

Daily recommended dosage of Vitamin E is $14-15 \mathrm{mg} /$ day [22.5 IU].

Sources: Eggs, Fortified cereals, Fruits, Green leafy vegetables (Spinach), Meat, Meat oils, Poultry, Vegetable oils.

Vitamin C helps in inhibition of peroxidation of membrane 
phospholipids, by improving the antimicrobial and NK cell activity, Chemotaxis, Lymphocyte proliferation, reduces Histamine [10].

A. Beta carotene: Increases activity of TNF- $\alpha$, stimulates NK cells, $\mathrm{T}$ helper cells andIL-2 receptor containing cells, immunomodulatory mechanism [1].

Lycopene has an antioxidant property, by stimulating Glutathione-s-transferase, Super oxide dismutase, and neutralizing the free radical's ROS release, inhibiting cell death, cell aging and inducing cell regeneration $[1,2]$.

B. Vitamin D: Active form of vitamin D is vitamin D3 $(1,25$ dihydroxycholecalciferol) also called as Calcitriol. Sun rays are natural source of Vitamin D inhibits prostaglandin synthesis, by suppressing the COX-2, NF-KB, MMP-2 inhibition, VEGF inhibition via HIF- $1 \alpha$ transcriptional factor and IL-8 inhibition via NF-KB down regulation [2].

Daily recommended dosage of Vitamin D is $600 \mathrm{IU} /$ day over 18 years of age.

Sources: Oily fish i.e., Salmon, Sardines, Red Meat, Liver, Mushroom, Egg yolk, Fortified foods.

Omega 3 fatty acids are Poly Unsaturated fatty acids and a key component of cell membrane Phospholipids. ALA, EPA, DHA are omega 3 fatty acids obtain from marine source. The ability of the body to form omega 3 fatty acids becomes lesser with age. Omega $\mathbf{3}$ fatty acids from dietary sources, improvesmuscle mass, reverse cancer cachexia, and promote weight maintenance. Alters EGFR, NF-Kb, MAPKs by signaling pathways and lipid peroxidation, which reverse cell damage [2].

Daily recommended dosage of Omega 3 fatty acids- $0.3-0.5$ gm/day.

Sources: Fish oil, Walnuts, Fish, Eggs, Soya Beans, Spinach, and Flax Seed oil.

Probiotics are live microorganisms that, when administered in adequate amounts, confer a health benefit on the host. It must contain a minimum 30x109CFU/gram.

They have an anti-inflammatory role and CRP(C-reactive protein) level reduction related to strain specific such as Lactobacillus, Bifidobacterium, Saccharomyces, Enterococcus, and E. coli. It decreases Cytokine signaling such as TNF- $\alpha$ and increases in TGF- $\beta$, IL-10 acts as an anti-inflammatory role, inhibition of inflammation by inactivating JAK2 and STAT1/STAT3 transcription factor activation. It inhibits pathogenic enteric bacterial growth by decreasing luminal PH by Lactic Acid. Lowers the mutagenic and toxigenic reaction by its metabolic product, protection of intestinal barrier integrity and increases the absorption of calcium and other minerals, decrease inflammation associated molecules by production of short chain fatty acids (butyrate and lactate) $[7,8]$.

Probiotics daily recommended dosages: 3x109 CFU

Sources: Yogurt, Milk, Diary products, and Soy beverages.

\section{Conclusion}

Anti-inflammatory dietary supplements prevent various diseases in geriatric peoples by suppressing inflammatory mediators such as free radicals (ROS, RNS), Cytokines, growth factors, transcription factors associated with cardiovascular and gastrointestinal disorders, including cancer. Improving overall patient outcome and survival.

\section{References}

1. Shrihari TG, Vijeev V, Soundarya K, Deepa Reddy, Manjunath V, et al. (2016) Anti-inflammatory dietary supplements in the chemoprevention oral cancer. Cancer research frontiers 2(3): 380-395.

2. Shrihari TG, Vijeev V, Kailasam S, Devaraju D, Manjunath V, et al. (2012) Antioxidants: Are we abusing it?. Journal of Indian academy of Oral medicine and radiology 24(4): 306-310.

3. Shrihari TG, Vasudevan V, Manjunath V, Devaraju D (2016) Potential co-relation between chronic periodontitis and cancer- An emerging concept. Gulf J Oncolog 1(20): 20-24.

4. Shrihari TG (2012) Potential Co-relation between periodontitis and coronary heart diseases- An overview. Gen Dent 60(1): 20-24.

5. Shrihari TG (2017) Dual role of inflammatory mediators in cancer. Ecancermedicalscience 11: 721.

6. Shrihari TG (2016) Inflammation related cancer- Highlights. J Carcinog Mutagen 7: 269-271.

7. Lescheid DW (2014) Probiotics as regulators of inflammation: A review. Functional Foods in Health and disease 4(7): 299-311.

8. Bermudez BM, Plaza DJ, Munoz QS, Gomez LC, Gi LA, et al. (2012) Probiotic mechanisms of action. Ann Nutr Metab 61(2): 160-174.

9. Satish BN, Dilipkumar P (2015) Free radicals, natural antioxidants, and their reaction mechanisms. RSC Adv 5: 27986-28006.

10. Maret GT, Jan FS (2011) Vitamin C and E: Beneficial effects from a mechanistic perspective. Free radic Biol med 51(5): 1000-1013. 